PREPARED FOR THE U.S. DEPARTMENT OF ENERGY, UNDER CONTRACT DE-AC02-76CH03073

PPPL-3762

PPPL-3762

UC-70

Eliminating Islands in High-pressure Free-boundary Stellarator Magnetohydrodynamic Equilibrium Solutions

by

S.R. Hudson, D.A. Monticello, A.H. Reiman, A.H. Boozer, D.J. Strickler, S.P. Hirshman, and M.C. Zarnstorff

November 2002

N/

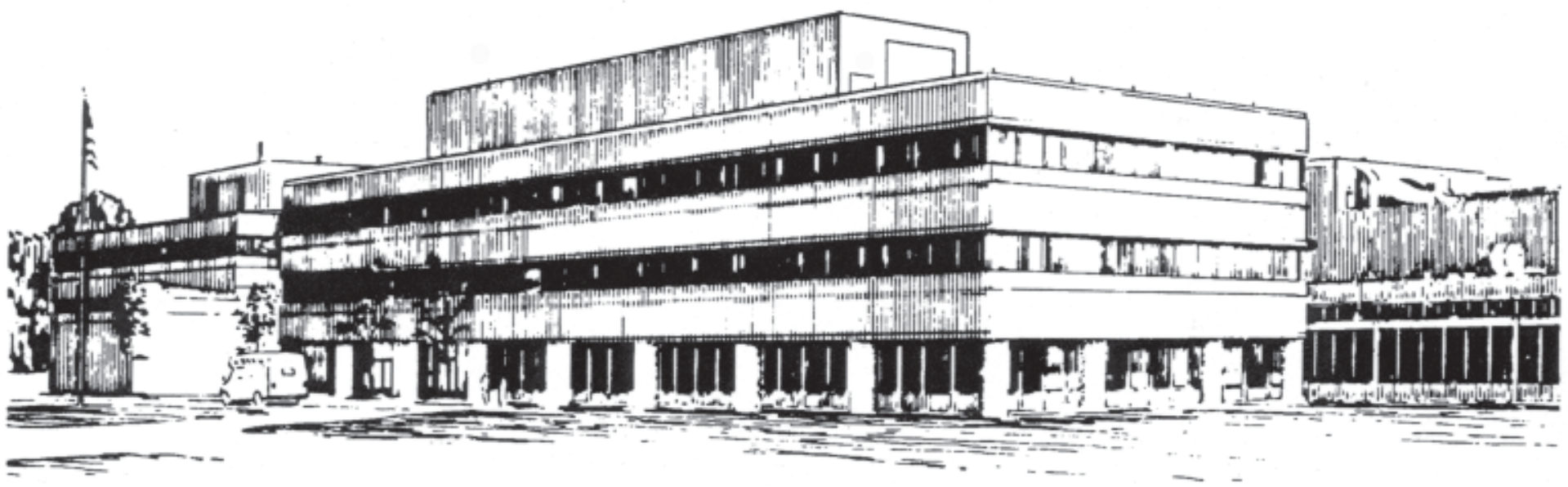

PRINCETON PLASMA PHYSICS LABORATORY PRINCETON UNIVERSITY, PRINCETON, NEW JERSEY 


\section{PPPL Reports Disclaimer}

This report was prepared as an account of work sponsored by an agency of the United States Government. Neither the United States Government nor any agency thereof, nor any of their employees, makes any warranty, express or implied, or assumes any legal liability or responsibility for the accuracy, completeness, or usefulness of any information, apparatus, product, or process disclosed, or represents that its use would not infringe privately owned rights. Reference herein to any specific commercial product, process, or service by trade name, trademark, manufacturer, or otherwise, does not necessarily constitute or imply its endorsement, recommendation, or favoring by the United States Government or any agency thereof. The views and opinions of authors expressed herein do not necessarily state or reflect those of the United States Government or any agency thereof.

\section{Availability}

This report is posted on the U.S. Department of Energy's Princeton Plasma Physics Laboratory Publications and Reports web site in Fiscal Year 2003. The home page for PPPL Reports and Publications is: http://www.pppl.gov/pub_report/

DOE and DOE Contractors can obtain copies of this report from:

U.S. Department of Energy

Office of Scientific and Technical Information

DOE Technical Information Services (DTIS)

P.O. Box 62

Oak Ridge, TN 37831

Telephone: (865) 576-8401

Fax: (865) 576-5728

Email: reports@adonis.osti.gov

This report is available to the general public from:

National Technical Information Service

U.S. Department of Commerce

5285 Port Royal Road

Springfield, VA 22161

Telephone: $1-800-553-6847$ or

(703) $605-6000$

Fax: (703) 321-8547

Internet: http://www.ntis.gov/ordering.htm 


\title{
Eliminating islands in high-pressure free-boundary stellarator magnetohydrodynamic equilibrium solutions.
}

\author{
S.R.Hudson ${ }^{1}$, D.A.Monticello ${ }^{1}$, A.H.Reiman ${ }^{1}$, A.H.Boozer ${ }^{2}$, D.J.Strickler ${ }^{3}$, S.P.Hirshman ${ }^{3}$, and M.C.Zarnstorff ${ }^{1}$ \\ 1) Princeton Plasma Physics Laboratory, P.O. Box 451, Princeton NJ 08543, USA. \\ 2) Columbia University, New York, New York 10027, USA. and \\ 3) Oak Ridge National Laboratory, P.O. Box 2009, Oak Ridge TN 37831, USA.
}

(Dated: November 7, 2002)

\begin{abstract}
Magnetic islands in free-boundary stellarator equilibria are suppressed using a procedure that iterates the plasma equilibrium equations and, at each iteration, adjusts the coil geometry to cancel resonant fields produced by the plasma. The coils are constrained to satisfy certain measures of engineering acceptability and the plasma is constrained to ensure kink stability. As the iterations continue, the coil geometry and the plasma simultaneously converge to an equilibrium in which the island content is negligible. The method is applied with success to a candidate plasma and coil design for the National Compact Stellarator eXperiment [Phys. Plas., 7:1911, 2000].
\end{abstract}

\section{INTRODUCTION}

The magnetic field lines of toroidal plasma confinement devices, such as stellarators [1], are $1 \frac{1}{2}$ dimensional Hamiltonian systems and magnetic flux-surfaces are the analog of constant action surfaces [2]. This may be seen by noting that in arbitrary toroidal coordinates $(r, \theta, \zeta)$ any vector, in particular the magnetic vector potential, may be written $\mathbf{A}=\psi \nabla \theta-\chi \nabla \zeta+\nabla g$, where $\psi, \chi$ and $g$ are functions of $(r, \theta, \zeta)$ : from which $\mathbf{B}=\nabla \psi \times \nabla \theta+\nabla \zeta \times \nabla \chi$. Using the toroidal angle $\zeta$ as the independent (time) coordinate, and considering $\chi=\chi(\psi, \theta, \zeta)$, the magnetic field line flow equations may be recast in a form identical to Hamilton's equations: $d_{\zeta} \theta=\partial_{\psi} \chi$ and $d_{\zeta} \psi=-\partial_{\theta} \chi$.

For magnetohydrodynamic (MHD) equilibrium, the pressure gradient force must balance the Lorentz force $\nabla p=\mathbf{J} \times \mathbf{B}$, which requires $\mathbf{B} \cdot \nabla p=0$. In regions where $|\nabla p| \neq 0$, the field is integrable, $\mathbf{B} \cdot \nabla \psi=0$, and action-angle coordinates exist $\chi=\chi(\psi)$. In this context, action-angle coordinates are called magnetic coordinates and $\mathbf{B} \cdot \nabla f=(\mathbf{B} \cdot \nabla \zeta)\left(t \partial_{\theta}+\partial_{\zeta}\right) f$ for an arbitrary function $f$ and $t=\partial_{\psi} \chi$ is called the rotational-transform.

Integrable $1 \frac{1}{2}$ dimensional Hamiltonians naturally occur only in systems with a continuous symmetry, and stellarators have no continuous symmetry. Integrability can be studied by perturbing an integrable field $\mathbf{B}_{\mathbf{0}}$. Writing $\mathbf{B}=\mathbf{B}_{0}+\mathbf{B}_{1}$ and $\psi=\psi_{0}+\psi_{1}$, the perturbed system is integrable if $\mathbf{B}_{0} \cdot \nabla \psi_{1}+\mathbf{B}_{1} \cdot \nabla \psi_{0}=0$. In magnetic coordinates this becomes

$$
t \frac{\partial \psi_{1}}{\partial \theta}+\frac{\partial \psi_{1}}{\partial \zeta}=-\frac{\mathbf{B}_{1} \cdot \nabla \psi_{0}}{\mathbf{B}_{0} \cdot \nabla \zeta}
$$

If this can be non-trivially solved for $\psi_{1}$, new magnetic coordinates exist and the perturbed state preserves integrability; however, the Fourier coefficients of $\psi_{1}$ are given by the Fourier coefficients of $\left(B_{1}^{\psi} / B_{0}^{\zeta}\right)$ divided by $(t m-n)$. At rational rotational-transform surfaces, $t=$ $n / m$, a singularity exists and the perturbed state is nonintegrable. In the perturbed state the rational surface splits to form an island of width $\left[\left(B_{1}^{\psi} / B_{0}^{\zeta}\right)_{m n} / t^{\prime} m\right]^{1 / 2}$.
Islands and the chaotic field lines caused by island overlap result in poor plasma confinement.

In an ideal MHD model of plasma perturbations, singular currents arise at the rational surfaces, both from $\delta$-function currents that arise to guarantee that islands can neither be created nor destroyed, and from singularities in the Pfirsch-Schlüter currents. The $\delta$-function currents cannot exist in a plasma equilibrium consistent with finite resistivity, and non-zero $\left(B_{1}^{\psi} / B_{0}^{\zeta}\right)_{m n}$ and thus islands may exist. In the presence of islands, the PfirschSchlüter currents are non-singular [3]. Numerical codes that model such equilibria, such as the PIES code [4], must allow for the magnetic field to have islands. Note that that there is an effect on the island width from the plasma currents in the island interior and near the separatrix (the apparent $\delta$-function currents as seen by the exterior solution in a boundary layer analysis [5]), and this effect is included in the PIES code.

Changes in coil geometry will change $\left(B_{1}^{\psi} / B_{0}^{\zeta}\right)_{m n}$ and can reduce the magnitude of the islands and their associated stochastic regions. It may not be possible to completely eliminate all islands [6], but all that is required in practice is that the magnetic islands occupy less than a tolerable percentage of the plasma volume. Such a magnetic field is said to have 'good-flux-surfaces'.

The construction of vacuum magnetic fields with goodflux-surfaces is not trivial [7], but is simpler than when a plasma is present. The additional complexity arises from the modification of $\left(B_{1}^{\psi} / B_{0}^{\zeta}\right)_{m n}$ by the plasma currents, and the self-consistent solution requires that the plasma equilibrium field and the coil field combine to give zero resonant component at the rational surfaces. Previous studies of finite pressure stellarator equilibria with islands have showed that the width of an island can depend on the magnitude of the plasma pressure and even go to zero, an effect called 'self-healing' [8]. A recent article [9] showed that high-pressure fixed-boundary solutions may be constructed with good-flux-surfaces.

Stellarators are designed to optimize both their physics properties (particle orbits, MHD stability, etc.) and the engineering of the coils. The optimizations rely on 
plasma equilibrium codes, and the fastest such codes presuppose perfect flux surfaces - the existence or size of magnetic islands cannot be addressed. The purpose of our study is to enforce good-flux-surfaces by varying the shape of the coils while preserving the optimized properties of the plasma and the coils. Stellarator coils must balance the normal field $B_{n}$ produced by the plasma currents on the plasma surface. Balancing $B_{n}$ at each point on an arbitrary surface represents an infinite number of constraints and generically leads to singular coil currents. Fortunately, each resonant $\left(B_{1}^{\psi} / B_{0}^{\zeta}\right)_{m n}$ that must be controlled constrains the magnitude of only one spatial distribution of $B_{n}$ on the plasma surface, and it is only this spatial distribution which must be nulled to eliminate the island.

The motivation for this work was the design of the National Compact Stellarator eXperiment (NCSX) [10]. Features of this design make the enforcement of goodflux-surfaces more difficult than in traditional stellarators [1]. NCSX is compact with a pronounced lack of geometric symmetry and has a large shear and transform per period, which produce multiple low order resonances. In addition NCSX is designed to operate with significant plasma current and at high plasma pressure (above $4 \%$ of the averaged magnetic energy), which means the rotational transform profile and the shape of the magnetic surfaces are equilibrium dependent.

We found: (1) Adjustments to the coil shapes allow the enforcement of good-flux-surfaces while maintaining optimized plasma and engineering properties of a particular NCSX equilibrium. (2) Coils obtained from healing a single reference configuration actually support many other optimized NCSX equilibria while maintaining good-fluxsurfaces. The second result indicates the primary issue with the NCSX flux surfaces is coil design and not the plasma equilibrium. The phenomenon of 'self-healing' implies this result is not generic, but the improved flux surfaces seen for a class of NCSX equilibria builds confidence that the method has practical as well as fundamental physical interest.

The free-boundary PIES code, which was used in the study, finds solutions by iterating the MHD equilibrium equations and has a representation of the magnetic field that accommodates magnetic islands and chaotic field lines. To suppress islands, the standard PIES algorithm is augmented so the coil geometry is altered at each iteration to cancel the resonant magnetic field components produced by plasma currents. The adjustment of the coils at each iteration allows the retention of the inherently non-linear plasma response. To preserve the previous optimization of the coils and the plasma, changes in the coil geometry are constrained to preserve engineering constraints of minimum bend radius and coil-coil separation, as well as the plasma constraint of ideal kink stability. As the iterations continue, the coil geometry and the plasma equilibrium simultaneously converge to an island-free, stable-plasma with build-able coils.

\section{METHOD}

The total magnetic field is the sum of the magnetic field produced by the plasma, $\mathbf{B}_{P}$, and the magnetic field produced by the confining coils, $\mathbf{B}_{C}$, which is a function of a set of Fourier harmonics, $\boldsymbol{\xi}$, which describe the coil geometry, at the $n$th PIES iteration

$$
\mathbf{B}^{n}=\mathbf{B}_{P}^{n}+\mathbf{B}_{C}\left(\boldsymbol{\xi}^{n}\right) .
$$

The initial plasma state is provided by the VMEC code [11], which imposes the artificial constraint that the plasma has nested flux-surfaces, and the initial coil geometry is provided by the COILOPT code [12]. The method presented in this article removes the constraint of nested surfaces and allows the VMEC initialization to relax into an equilibrium, potentially with broken fluxsurfaces (islands), while making adjustments to the coil set to remove selected islands as they develop.

The PIES iterations solve for the plasma current $\mathbf{J}$ given $\mathbf{B}$ and given pressure profile $p$

$$
\nabla p=\mathbf{J}^{n+1} \times \mathbf{B}^{n} .
$$

A magnetic-differential equation $\mathbf{B} \cdot \nabla\left(J_{\|} / B\right)=\nabla \cdot J_{\perp}$ gives the parallel current which is solved using magnetic coordinates [13], and the current profile enters as an integration constant. PIES uses current profiles which are consistent with an Ohm's law with finite resistivity, thus eliminating the $\delta$-function parallel currents. The PIES code allows the field topology to break up into islands and chaos. In the region interior to the islands, because of thermal and particle diffusion and in the absence of sources and sinks, the pressure is constant and thus the magnetic differential equation for the PfirschSchlüter currents need not be solved. Also, to be consistent with Ohm's and Faraday's laws in steady state, the current profile is flattened inside the island.

The plasma magnetic field is then solved given $\mathbf{J}$, and blended to provide numerical stability :

$$
\begin{aligned}
\mathbf{J}^{n+1} & =\boldsymbol{\nabla} \times \mathbf{B}_{P}, \\
\mathbf{B}_{P}^{n+1} & =\alpha \mathbf{B}_{P}^{n}+(1-\alpha) \mathbf{B}_{P} .
\end{aligned}
$$

Typically the blending parameter $\alpha=0.99$ for NCSX style equilibria. The standard PIES algorithm makes no changes to the coil geometry and iterates through equations $(3,4,5)$ to calculate the free-boundary equilibrium for a given pressure profile and coil set.

The additional steps in the implementation of the coilhealing are as follows. The total magnetic field $\overline{\mathbf{B}}$ is

$$
\overline{\mathbf{B}}=\mathbf{B}_{P}^{n+1}+\mathbf{B}_{C}\left(\boldsymbol{\xi}^{n}\right) .
$$

We may consider $\overline{\mathbf{B}}$ as a nearly integrable field and that magnetic islands are caused by fields normal to and resonant with rational rotational-transform flux-surfaces of a nearby integrable field. 
A set of resonances that are to be suppressed is selected. The selection is determined by the rotationaltransform profile. Islands associated with low-order rationals are typically the largest, but where the shear is small high-order islands can easily overlap and result in chaotic field lines. A set of toroidal surfaces matching the selected resonances is constructed. Each such surface (a quadratic-flux-minimizing surface [14]) may be considered as a rational rotational-transform flux-surface of an underlying integrable field [15], with each surface passing directly through its associated island chain and containing the stable and unstable periodic orbits. The construction of the quadratic-flux-minimizing surfaces provides an optimal magnetic coordinate system, or equivalently an optimal nearby integrable magnetic field, and in these coordinates resonant perturbation harmonics are clearly identified. The method is computationally efficient as the quadratic-flux-minimizing surfaces are constructed exactly and only where required - at the rational rotational-transform surfaces where islands develop.

The amplitude of each of the $N$ selected resonant field harmonics, denoted $\left\{\bar{B}_{i}: i=1, N\right\}$, is calculated by Fourier decomposing the magnetic field normal to the quadratic-flux-minimizing surface. The Fourier decomposition is performed using an angle coordinate which corresponds to a magnetic coordinate angle of the underlying integrable field on that surface.

The COILOPT [12] code provides a convenient Fourier representation of the coil geometry and a set of $M$ coil harmonics $\left\{\xi_{j}: j=1, M\right\}$ is systematically varied to set $\bar{B}_{i}=0$ using a Newton method. The coupling matrix, $\nabla B_{C i j}^{n}$, is defined as the partial derivatives of the selected resonant harmonics of the coil magnetic field normal to the quadratic-flux-minimizing surface, which is updated every PIES iteration, with respect to the chosen coil harmonics and is calculated using finite-differences. A multi-dimensional Newton method is applied to find the coil changes $\delta \xi_{j}$ that set $\bar{B}_{i}=0$

$$
-\bar{B}_{i}=\sum_{j} \nabla B_{C i j}^{n} \cdot \delta \xi_{j}^{n} .
$$

This equation is solved for the $\delta \xi_{j}$ in a few iterations by inverting the $N \times M$ matrix $\nabla B_{C i j}^{n}$ using singular-value decomposition [16] and the coil set is adjusted

$$
\xi_{j}^{n+1}=\xi_{j}^{n}+\delta \xi_{j}^{n},
$$

at every PIES iteration, such that resonant components of the combined plasma-coil field are eliminated. As the iterations proceed, the coil geometry and the plasma simultaneously converge to coil geometry-plasma solution with good-flux-surfaces.

To be 'build-able', the minimum coil-curvature and coil-coil separation, for example, of the coils must exceed certain limits. Such constraints are calculated by the COILOPT code and the initial coil set, described by $\boldsymbol{\xi}^{0}$, is satisfactory from an engineering perspective. The healing algorithm is modified to preserve the minimum curvature and coil-coil separation by adding to the set of resonant fields to be eliminated the (appropriately weighted) differences in minimum curvature and coil-separation of the $n$th coil set, described by $\boldsymbol{\xi}^{n}$, from the initial coil set. This constrains the island-eliminating coil variations to lie in the nullspace of these measures of engineering acceptability. In a similar manner, the algorithm preserves kink stability. The VMEC initialization is kink-stable, and kink stability is calculated with the TERPSICHORE code [17].

\section{APPLICATION TO NCSX}

The method is routinely applied to NCSX [10] candidate coil and plasma designs. NCSX is a proposed proof-of-principle device with three field periods, aspect ratio $A=4.4$, major radius $\mathrm{R}=1.4 \mathrm{~m}$ and magnetic field $\mathrm{B}=1.7 \mathrm{~T}$. The stellarator symmetric coil design consists of 18 modular coils ( 3 distinct coil types), 18 toroidal field coils, and six pairs of poloidal field coils and some additional trim coils. The plasma is designed to be quasiaxisymmetric to give good transport, and is stable to kink modes at $\beta \sim 4 \%$, but is marginally unstable to infinite-n ballooning modes. The rotational-transform profile has $t \sim 0.4$ on axis, maximum $t \sim 0.66$ near the edge and $t \sim 0.65$ at the edge: including the low order resonances $t=3 / 7,3 / 6$ and $3 / 5$. Note that the shear vanishes near the $t=6 / 9$ resonance.

Considering a candidate coil set and selecting the $(n, m)=(3,6),(3,5)$ islands to be suppressed, subject to the constraint that the minimum coil curvatures, the coil-coil separation and the kink stability be preserved (9 constraints), and allowing some $m=3,4,5,6,7,8$ modular coil harmonics to vary (36 independent variables), a healed coil-plasma state is achieved. The engineering measures are preserved and the plasma is stable with respect to kink modes. Also, the plasma retains quasiaxisymmetry and is stable to ballooning modes $n<45$.

Several hundred iterations are required to approach convergence in both the plasma field and the coil geometry. To confirm convergence several hundred additional standard PIES iterations are performed with the coil set unchanged. A Poincaré plot of the final field is shown on an up-down symmetric toroidal cross-section in the upper half of Fig. 1. The island content in the healed configuration is negligible, though there is some resonant $m=18$ deformation near the zero shear location and some high order ( $m=10,11,12$, and 14) island chains. For comparison, a Poincaré plot of the unhealed configuration is shown after 180 standard PIES iterations in the lower half of Fig. 1. For the unhealed case there is a large $m=5$ island and the configuration deteriorates into large regions of chaos.

The maximum coil alteration is about $2 \mathrm{~cm}$, which comfortably exceeds manufacturing tolerances, but is not so large that 'healing' significantly impacts other design concerns, such as diagnostic access. The coil harmonics varied actually describe the toroidal variation of the 
modular coils on a toroidal winding surface. The calculation shown used 63 radial surfaces, 12 poloidal and 6 toroidal modes. Similar results have been obtained using up to 93 radial surfaces and 20 poloidal modes.

\section{COMMENTS}

The flux-surface quality of the 'healed' equilibrium shows remarkable improvement compared to the unhealed configuration. The coils have been described with a filamentary model, and a finite thickness model of the healed coils shows further improvement, in particular the $m=18$ deformation and the high order islands are reduced. To model a discharge evolution, we considered a sequence of equilibria with increasing plasma pressure. Though islands may re-appear as the configuration departs from the healed configuration, the island content in each of the equilibria with the healed coils is much smaller than in the corresponding equilibrium with the original coils.

In principle, in the limit of suppressing additional islands, this approach can lead to non-axisymmetric coil- plasma configurations with integrable magnetic fields. The procedure amounts to a stellarator design optimization routine that for the first time provides a mechanism for suppressing magnetic islands, while providing ideal stability and satisfying engineering constraints. In addition to the improvement in particle confinement associated with good-flux-surfaces, the construction of integrable fields has implications for stellarator MHD stability calculations, which are usually based on equilibria artificially constrained to have nested flux-surfaces. As the equilibria constructed using this method, and the method presented in [9], relax the unphysical imposition of nested surfaces, but nevertheless maintain integrability by careful design, stability studies based on these equilibria are expected to be more reliable.

We thank the NCSX design team, Long-Poe Ku and Guo-Yong Fu for stability analysis, Raul Sanchez and Tony Cooper for use of the COBRA and TERPSICHORE codes, and to Amitava Bhattacharjee for advice regarding the manuscript. This work was supported in part by US Department of Energy contract DE-AC02$76 \mathrm{CH} 03073$.
[1] A.H. Boozer. Phys. Plas., 5(5):1647, 1998;

[2] J.R. Cary and R.G. Littlejohn. Annals of Physics 151:1, 1983; A.H. Boozer. Phys. Plas., 26(4):1288, 1983.

[3] A.H.Reiman, N.Pomphrey and A.H.Boozer. Phys. Flu. B., 1(3):555, 1989.

[4] A.H. Reiman and H.S. Greenside. Comp. Phys. Comm., 43:157, 1986.

[5] A. Bhattacharjee, T. Hayashi et al. Phys. Plas., 2(3):883, 1995 ;

[6] H. Grad. Phys. Flu. 10(1):137, 1967.

[7] J.R. Cary. Phys. Rev. Lett., 49(4):276, 1982; S.R. Hudson and R.L. Dewar. Phys. Lett. A, 226:85, 1997.

[8] T. Hayashi et al. Phys. Plas. 1(10):3262, 1994. S.S. Lloyd, H.J. Gardner et al. Journal of Plasma and Fusion Research SERIES, 1:484, 1997.

[9] S.R. Hudson, D.A. Monticello, and A.H. Reiman. Phys. Plas., 8(7):3377, 2001.
[10] Reiman et al. Phys. Plas. 8(5):2083, 2001.

[11] S.P. Hirshman and O. Betancourt. J.Comp. Phys., 96:99, 1991.

[12] D.J. Strickler, L.A. Berry, and S.P. Hirshman. Fusion Science and Technology, 41, 2001.

[13] A.H. Reiman and H.S. Greenside. J.Comp. Phys., 75(2):423, 1988.

[14] R.L. Dewar, S.R. Hudson, and P. Price. Phys. Lett. A, 194:49, 1994.

[15] S.R. Hudson and R.L. Dewar. Phys. Plas., 6(5):1532, 1999.

[16] W.H. Press, B.P. Flannery et al. Numerical Recipes in Fortran 77: The art of scientific computing. Cambridge University Press, Cambridge, U.K., 2nd edition, 1992.

[17] W.A. Cooper, Plasma Phys. Controlled Fusion 34(6):1011 (1992). 
FIG. 1: Poincaré plot of the converged healed coil-plasma field (upper) and for the original, unhealed coils after 180 standard PIES iterations (lower) for the NCSX candidate coil set M45. The VMEC initialization boundary is shown as the thick solid line.

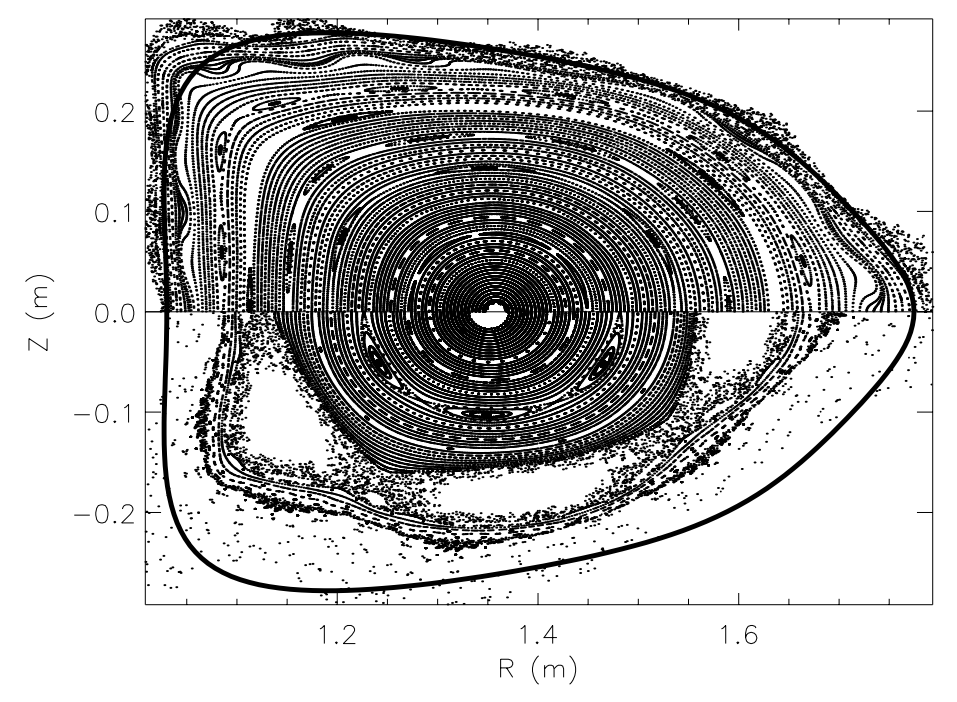




\section{External Distribution}

Plasma Research Laboratory, Australian National University, Australia

Professor I.R. Jones, Flinders University, Australia

Professor João Canalle, Instituto de Fisica DEQ/IF - UERJ, Brazil

Mr. Gerson O. Ludwig, Instituto Nacional de Pesquisas, Brazil

Dr. P.H. Sakanaka, Instituto Fisica, Brazil

The Librarian, Culham Laboratory, England

Library, R61, Rutherford Appleton Laboratory, England

Mrs. S.A. Hutchinson, JET Library, England

Professor M.N. Bussac, Ecole Polytechnique, France

Librarian, Max-Planck-Institut für Plasmaphysik, Germany

Jolan Moldvai, Reports Library, MTA KFKI-ATKI, Hungary

Dr. P. Kaw, Institute for Plasma Research, India

Ms. P.J. Pathak, Librarian, Insitute for Plasma Research, India

Ms. Clelia De Palo, Associazione EURATOM-ENEA, Italy

Dr. G. Grosso, Instituto di Fisica del Plasma, Italy

Librarian, Naka Fusion Research Establishment, JAERI, Japan

Library, Plasma Physics Laboratory, Kyoto University, Japan

Research Information Center, National Institute for Fusion Science, Japan

Dr. O. Mitarai, Kyushu Tokai University, Japan

Library, Academia Sinica, Institute of Plasma Physics, People's Republic of China

Shih-Tung Tsai, Institute of Physics, Chinese Academy of Sciences, People's Republic of China

Dr. S. Mirnov, TRINITI, Troitsk, Russian Federation, Russia

Dr. V.S. Strelkov, Kurchatov Institute, Russian Federation, Russia

Professor Peter Lukac, Katedra Fyziky Plazmy MFF UK, Mlynska dolina F-2, Komenskeho Univerzita, SK-842 15 Bratislava, Slovakia

Dr. G.S. Lee, Korea Basic Science Institute, South Korea

Mr. Dennis Bruggink, Fusion Library, University of Wisconsin, USA

Institute for Plasma Research, University of Maryland, USA

Librarian, Fusion Energy Division, Oak Ridge National Laboratory, USA

Librarian, Institute of Fusion Studies, University of Texas, USA

Librarian, Magnetic Fusion Program, Lawrence Livermore National Laboratory, USA

Library, General Atomics, USA

Plasma Physics Group, Fusion Energy Research Program, University of California at San Diego, USA

Plasma Physics Library, Columbia University, USA

Alkesh Punjabi, Center for Fusion Research and Training, Hampton University, USA

Dr. W.M. Stacey, Fusion Research Center, Georgia Institute of Technology, USA

Dr. John Willis, U.S. Department of Energy, Office of Fusion Energy Sciences, USA

Mr. Paul H. Wright, Indianapolis, Indiana, USA 
The Princeton Plasma Physics Laboratory is operated by Princeton University under contract with the U.S. Department of Energy.

\author{
Information Services \\ Princeton Plasma Physics Laboratory \\ P.O. Box 451 \\ Princeton, NJ 08543
}

Phone: 609-243-2750

Fax: 609-243-2751

e-mail: pppl_info@pppl.gov

Internet Address: http://www.pppl.gov 